\title{
Experiencia de voluntariado de salud mental en estudiantes de enfermería. Una aproximación cualitativa
}

An Experience in Mental Health Volunteering among Nursing Students. A qualitative approach

\section{Myriam Rodríguez Miguel', Rocio Juliá-Sanchis², Ángela Pérez Esquerdo ${ }^{3}$, Verónica Pérez Esquerdo', Marcelino Pastor-Bernabeu²}

\author{
${ }^{1}$ Estudiante de Grado de Enfermería. Facultad de Ciencias de la Salud, Universidad de Alicante. Alicante, España \\ ${ }^{2}$ Departamento Enfermería, Facultad de Ciencias de la Salud. Universidad de Alicante. Alicante, España. \\ ${ }^{3}$ Hospital Universitario San Juan. Alicante, España. \\ Contacto: myriam-altea@hotmail.com \\ Fecha de recepción: 22 de junio de 2018 / Fecha de aceptación: 09 de octubre de 2018
}

\begin{abstract}
Resumen
Introducción: El estigma y los prejuicios hacia la salud mental existen tanto entre la población general como entre los profesionales de la salud. La participación de estudiantes en diferentes actividades en el ámbito clínico de la salud mental puede llegar a modificar las actitudes y reducir el estigma asociado a la enfermedad.

Objetivo: Conocer la experiencia de estudiantes de Enfermería tras participar en un voluntariado de salud mental y conocer el cambio de actitudes desencadenado de dicha experiencia.

Métodos: Este estudio utilizó un diseño cualitativo descriptivo que incluyó entrevistas individualizadas a nueve estudiantes de enfermería de cuarto grado de la Universidad de Alicante que participaron en un voluntariado de salud mental realizado en el Hospital Universitario de San Juan (Alicante).

Resultados: A través del análisis de las entrevistas se generaron seis temas. El tema "Experiencias previas" describe las vivencias que las participantes habían tenido con relación a la salud mental. El tema "Sentimientos" recoge todos los sentimientos experimentados por las participantes tanto antes como después del voluntariado. El tema "Aprendizaje" abarca todo aquello que las participantes creen que han obtenido de su paso por el voluntariado. El tema "Valor del voluntariado" engloba todos aquellos aspectos que determinaron la importancia que las entrevistadas dieron a su participación en el voluntariado. El tema "Enfermería de salud mental" contempla la opinión sobre algunos de las participantes sobre cómo trabajan las enfermeras en la unidad de hospitalización. El último tema, "Describiendo experiencias", recoge las experiencias y las anécdotas que los estudiantes vivieron durante el voluntariado.

Conclusiones: Los estudiantes obtienen beneficios de la participación en voluntariados de salud mental. No solo se describen las positivas experiencias, sino que también se pone de manifiesto un cambio de actitud entre las participantes, desmontando los prejuicios y las ideas preconcebidas con las que contaban antes de su participación.
\end{abstract}

Palabras clave: estudiantes, enfermería, salud mental, voluntariado.

\begin{abstract}
Introduction: The stigma and prejudices towards mental health exist both among the general population and among health professionals. The participation of students in different activities in the clinical field of mental health can change attitudes and reduce the stigma associated with the disease.

Aim: To know the experience of nursing students in a mental health volunteer and to know the change of attitudes unchained from this experience.

Methods: This study used a qualitative descriptive design that includes individualized interviews to nine fourthdegree nursing students of the University of Alicante who participate in a mental health volunteer that takes place in the University Hospital of San Juan (Alicante).

Findings: Six themes were generated through the analysis of the interviews. The theme "Previous Experiences" describes the experiences that participants have had in relation to mental health. The "Sentiments" theme collects all the feelings experienced by the participants before and after volunteering. The "Learning" theme covers everything attendees believe they have achieved through volunteering. The theme "Volunteering value" encompasses all those aspects that determined the importance that the interviewees gave to their participation in the voluntary work. The
\end{abstract}


subject "Mental Health Nursing" contemplates the opinion on some of the participants about how nurses work in the hospitalization unit. The last topic, "Describing Experiences," collects the experiences and anecdotes that the students lived during volunteering.

Conclusions: The students obtained benefits from participation in mental health volunteering. Not only are described the positive experiences, but also is described a change among the participant's attitude, moving away the prejudices and the preconceived ideas that they had before their participation.

Keywords: mental health, students, nursing, volunteering.

\section{Introducción}

Los trastornos mentales tienen un gran impacto en la población europea, siendo el estigma una de las principales causas de dicho impacto ${ }^{1}$.

La estigmatización es un constructo social que incluye actitudes, sentimientos, creencias y comportamientos que se configuran como prejuicios, lo que supone consecuencias discriminatorias hacia las personas con algún trastorno mental ${ }^{2}$. Estas creencias negativas no existen únicamente en la población general, sino que también los profesionales y los estudiantes de Enfermería participan en este proceso de estigmatización ${ }^{3}$. La falta de conocimientos sobre las enfermedades mentales y la salud mental en general, unido a las falsas creencias existentes entre la población general, conlleva la discriminación ${ }^{4}$.

La existencia de estigma entre los profesionales de la salud y los estudiantes puede influir de manera significativa en la salud y la calidad de vida de los pacientes y sus familiares, facilitando el desarrollo de cuadros depresivos, mayor ansiedad, aislamiento social y disminución de la autoestima, lo que supone una afectación negativa a las tasas de recuperación ${ }^{4,5}$.

Varios estudios sugieren que hay un cambio significativo de actitudes hacia la enfermedad mental y la enfermería psiquiátrica por parte de los estudiantes de Enfermería tras realizar sus prácticas clínicas en salud mental, partiendo generalmente de actitudes negativas que se transforman en positivas tras la realización de las prácticas ${ }^{3}$. Del mismo modo, según en un estudio realizado en Corea del Sur ${ }^{6}$, los estudiantes de Enfermería tuvieron una impresión negativa de la psiquiatría antes de llevar a cabo las prácticas hospitalarias. Los programas de actividades, programas de rehabilitación y programas terapéuticos realizados en el ámbito de psiquiatría ayudaron a eliminar los prejuicios existentes en los estudiantes, cambiando completamente su actitud con respecto a la salud mental. Por tanto, la participación de los estudiantes de Enfermería en pro- gramas y actividades en el ámbito clínico, más allá de las prácticas realizadas de manera obligatoria a lo largo de su formación, reduce el estigma asociado a la enfermedad. Asimismo, la adquisición de experiencia y conocimientos produce grandes modificaciones, no solo en la postura de los estudiantes hacia la enfermedad, sino también hacia el campo de la salud mental, sintiéndose más preparados para trabajar en este ámbito en su futuro profesional ${ }^{7.8}$. De esta manera, además, se consigue reducir el temor y la ansiedad sufridos por los estudiantes antes de la realización de las prácticas clínicas, asociados al desconocimiento y la falta de habilidades de comunicación con personas con algún trastorno mental ${ }^{9}$.

El objetivo de este trabajo fue conocer la experiencia de estudiantes de Enfermería tras formar parte de un voluntariado de salud mental en una unidad de agudos, y conocer el cambio de actitudes desencadenado de dicha experiencia.

\section{Métodos}

En este estudio de método cualitativo, se utilizó un muestreo de conveniencia. Se solicitó la participación en una iniciativa de voluntariado de salud mental que se realizaba en la Unidad de Hospitalización Psiquiátrica (UHP) del Hospital Universitario de San Juan Alicante, a 199 estudiantes de cuarto curso del Grado de Enfermería. De estos, 40 (20\%) aceptaron participar. El tamaño de muestra se determinó cuando se alcanzó un punto de saturación de los datos adquiridos, 9 participantes. Las entrevistas semiestructuradas fueron grabadas, transcritas textualmente y analizadas por tres investigadores, lo que produjo un análisis de datos triangulados.

Todas las participantes fueron informadas por el investigador principal sobre el objetivo del estudio, los métodos utilizados y su forma de participación. Antes de ser entrevistadas, se obtuvo el consentimiento informado por escrito de las participantes. Las características definitorias de la muestra se representan en la Tabla 1. 


\begin{tabular}{|l|l|l|l|l|l|}
\hline Identificación & Edad & Género & $\begin{array}{l}\text { Contacto } \\
\text { previo }\end{array}$ & Tipo de actividad & $\begin{array}{l}\mathbf{N}^{0} \text { de sesiones en } \\
\text { las que participó }\end{array}$ \\
\hline E1 & 21 & Mujer & Sí & Juegos de mesa & 2 veces \\
\hline E2 & 21 & Mujer & No & Juegos; manualidades & 2 veces \\
\hline E3 & 21 & Mujer & Sí & Manualidades & 1 vez \\
\hline E4 & 23 & Mujer & No & Juegos; manualidades & 2 veces \\
\hline E5 & 31 & Mujer & Sí & Juegos & 3 veces \\
\hline E6 & 21 & Mujer & Sí & Actividades en huerto & 3 veces \\
\hline E7 & 22 & Mujer & Sí & Juegos & 4 veces \\
\hline E8 & 21 & Mujer & No & Lectura; juegos & 2 veces \\
\hline E9 & 27 & Mujer & Sí & Manualidades & 3 veces \\
\hline Media & 23,1 & - & - & - & 2,4 \\
\hline
\end{tabular}

Tabla 1. Características de la población.

\section{Procedimiento}

Los estudiantes de pregrado fueron informados durante las clases de teoría sobre la intervención propuesta, lo que implicó su apoyo y colaboración en los talleres de arteterapia en la UHP. Los posibles participantes se presentan voluntariamente al profesorado responsable que los invitó a asistir a una reunión en la que se les informó ampliamente.

Los estudiantes de enfermería se registraron como voluntarios oficiales del servicio de voluntariado "Humanización" del hospital donde se llevó a cabo la actividad. Recibieron una tarjeta de identificación y recibieron información sobre las reglas del servicio voluntario. Desde octubre de 2017 hasta marzo de 2018, mensualmente, los voluntarios participaron en sesiones de terapia artística y realizaron talleres previamente preparados bajo la supervisión del profesorado y los profesionales de la unidad.

\section{Recopilación de datos}

Los datos relevantes se recopilaron a través de entrevistas semiestructuradas realizadas en febrero de 2018. Los campos que se pretendían captar se encuentraban insertados en la formulación de diez preguntas abiertas, generadas a partir de la revisión bibliográfica y la formulación del objetivo, con la finalidad de que las participantes expresaran sus opiniones y vivencias acerca del tema principal. El esquema de entrevista se presenta a continuación:
- ¿Habías tenido trato previo con personas con algún problema de salud mental?

- ¿Cuántas veces has ido al voluntariado?

- ¿Qué tipo de actividades preparasteis?

- ¿Era la primera vez que visitabas una unidad de agudos?

- ¿Qué te pareció?

- ¿Qué sentimientos experimentaste?

- ¿Crees que trabajar en salud mental, es más peligroso que trabajar en otra unidad?

- ¿Crees que este voluntariado ha ayudado a los pacientes?

- ¿Cómo crees que ha cambiado, a través de tu paso por el voluntariado, tu visión sobre la salud mental y sobre los pacientes?

- ¿Cómo valorarías la experiencia de este voluntariado?

Una vez que las participantes fueron seleccionadas, un miembro del equipo de investigación las contactó por el WhatsApp del grupo de estudiantes. Establecido el contacto, se explicaron las características del estudio y se las invitó a participar. Las entrevistas se realizaron en aulas propias de la Universidad de Alicante, donde se logró un nivel apropiado de privacidad y confidencialidad. El momento de la cita se ajustó a las preferencias de las participantes. Las entrevistas duraron aproximadamente 20 minutos. 


\section{Análisis de los datos}

Los investigadores garantizaron la coherencia y precisión de los datos con respecto a la realización de entrevistas. Las entrevistas se grabaron con un dispositivo de grabación digital, se transcribieron textualmente, y posteriormente se presentaron a las participantes para corroborar la precisión de la transcripción. Las transcripciones fueron codificadas asignándoles la letra " $E$ " y un número según el orden cronológico en el que se realizaron (E1, E2, E3..., E9). Los datos se procesaron mediante un enfoque de análisis de contenido cualitativo $^{10}$. Las entrevistas fueron analizadas por los autores a través de la triangulación de datos, aplicando un sistema de codificación abierto e inductivo que consistía en la asignación de códigos emergentes a cada párrafo o frase, que resumía su significado. Estos códigos se clasificaron en grupos de acuerdo con la similitud. Posteriormente a la identificación de patrones en las transcripciones de las entrevistas, las clasificaciones se dividieron en temas y subtemas. Una vez identificadas, analizadas y contrastadas las posibles diferencias con respecto a la bibliografía y/o marcos conceptuales disponibles, el contenido fue luego examinado y se llegó a un consenso sobre los datos más relevantes que conformaban cada tema y subtema. La confiabilidad de los datos cualitativos se logró a través del proceso sistemático de recopilación y análisis de datos ${ }^{11}$.

\section{Resultados}

Las 9 personas entrevistadas tenían, en promedio, 23.1 años de edad $(\mathrm{DE}=3,9)$, siendo es su totalidad mujeres. El $60 \%$ afirmó haber tenido experiencia previa con personas con algún problema de salud mental antes de contribuir a este estudio como voluntarias, en su mayor parte como miembros de la familia $(70.0 \%)$, seguido por amigos y pacientes.

A través del análisis de las entrevistas, se generaron tres temas y once subtemas (ver Tabla 2).

\begin{tabular}{l|l} 
Temas & Subtemas \\
Experiencias previas & - Familiares \\
& - Pacientes \\
& - Sin experiencia \\
& - Miedo \\
Sentimientos y actitudes iniciales & - Sorpresa/ desconcierto \\
& - Actitudes negativas \\
& - Aprendizaje \\
& - Cambio de actitudes en las participantes \\
& - Experiencia personal \\
& - Aportaciones a los pacientes \\
& - Esto no son prácticas
\end{tabular}

Tabla 2. Relación de temas y subtemas identificados.

\section{Tema 1. Experiencias previas.}

Se incluyeron los siguientes subtemas: "Familiares", "Pacientes" y "Sin experiencia". La experiencia previa al voluntariado puede determinar las expectativas y/o percepciones de los estudiantes sobre los pacientes y las unidades de salud mental, por lo que se analizaron las vivencias que los encuestados habían tenido en relación a este tema.
Cuatro de las participantes tenían familiares cercanos con algún trastorno mental: "Mi padre tiene depresión desde hace muchos años" (E7), o: "Mi tía tiene trastorno bipolar" (E6). Otra estudiante explicaba: "Tengo a mi tía en casa, tiene discapacidad intelectual, y también tengo un primo con Síndrome de Down [...] Es que en mi familia hay bastantes cosas" (E1). En el caso de otro participante, la relación familiar era más lejana: "El hermano de mi cuñado tiene un diagnóstico y alguna persona más de mi familia” (E5). 
Sin embargo, para una entrevistada (E3), el contacto se basó en su experiencia durante las prácticas clínicas obligatorias del Grado de Enfermería, tanto en el contexto comunitario: "Estando de prácticas por la noche, hubo una urgencia: ir a casa de una chica con un brote psicótico, que ya sufría esquizofrenia", como en el contexto hospitalario: "Cuando estuve en Medicina Interna de prácticas, mi tutora la llamaron para pinchar una medicación. Entonces, fui con ella, pero solo miré, no interactué con nadie ni nada".

Las entrevistadas coincidieron en remarcar que esa experiencia vital hizo que no tuvieran tanto temor de las personas que conocerían en la UHP.

Sin embargo, para los que: "Antes de asistir a este voluntariado, no había tenido ningún contacto" (E2), no disfrutaron de esa ventaja.

\section{Tema 2. Sentimientos y actitudes iniciales.}

Este tema recoge las actitudes y los sentimientos experimentados por los estudiantes antes y durante el voluntariado. Se identificaron principalmente tres: "Miedo", "desconcierto/sorpresa" y "prejuicios".

Algunos de las entrevistadas experimentaron miedo relacionado con el desconocimiento y la falta de experiencia como así lo expresó esta alumna (E7): “Al principio un poco de miedo porque se acercaban y no sabía cómo ponerme o qué decir".

Sin embargo, la mayoría de las participantes resaltaron que sintieron miedo al ver por primera vez los sistemas de seguridad de la planta de hospitalización y su estructura, como así lo relataron dos participantes: "El primer contacto, cuando llegas a la planta y ves toda esa seguridad, lo de ir cerrando las puertas [...] A nosotros nos impresionó, a mí me asustó un poco por el hecho ese, de por qué tienen que cerrar todo tanto" (E1); y continua diciendo otra: "Cuando entras te da un poco de miedo porque ves un cartel que pone: 'Cierren bien la puerta por su seguridad'". Así mismo, una tercera estudiante experimentó una sensación similar: "Cuando vi la puerta, de verdad que sentí un poco de miedo, porque encima había dos pacientes paseando por los pasillos y era como un poco de respeto, ¿no?” (E4).

No obstante, para otras dos estudiantes no fue miedo, sino desconcierto y/o sorpresa los sentimientos que afloraron al conocer por primera vez al ver la infraes- tructura de la unidad. Por ejemplo, E2 explicó: “A1 entrar en la planta, era un servicio cerrado con dos puertas en el que para acceder tenías que o usar tu tarjeta o llamar al timbre [...] lo vi todo como muy protegido [...] Y como tampoco he estado allí de prácticas, tampoco sabía bien cómo se organizan o el porqué de todo eso"; y otra compañera dijo: "Un poco raro porque casi todas las puertas estaban cerradas y no es como en otras unidades que están las familias y los enfermos" (E5).

Las ideas preconcebidas negativas estuvieron presentes en la mayoría de las participantes antes de su paso por el voluntariado, incluso en aquellas participantes que tenían experiencias previas: "Hay algunas que se les nota la enfermedad, pero hay otras que no..." (E3), o: "Nos lo imaginábamos peor" (E6). Y otra estudiante explicaba: "Entró un hombre y se sentó y después entró otro. Propusieron jugar al ajedrez entre ellos y nosotras estábamos mirando y de repente, pues el señor que entró más tarde se puso a hablarle con una entonación más alta y nos miramos diciendo: 'Uy, ¿qué va a pasar?' [...] en ese momento se levantó, tiró la silla y las fichas y yo pensé: 'A ver si se le va un poco la pinza y nos hace algo...'. Pero no, después se fue, se calmó y cuando vino súper bien, estuvo jugando al parchís y todo y bien, pero al principio... fatal" (E4).

\section{Tema 3: Valor del voluntariado.}

En este tema se englobaron todos aquellos aspectos que determinaron la importancia que las entrevistadas dieron a su participación en el voluntariado. A partir de este tema se desarrollaron los subtemas: "Aprendizaje", "Cambio de actitudes en las participantes", "Experiencia personal", "Aportaciones a los pacientes" y "Esto no son prácticas".

Las participantes refirieron haber aprendido tras su paso por el voluntariado. El hecho de haber podido reforzar los conocimientos teóricos sobre salud mental y aplicarlos, facilitó su integración: "Al salir hablábamos de las personas que habíamos conocido e intentábamos ver si con lo que habíamos dado en clase de teoría nos cuadraba su comportamiento" (E6). "Comentábamos un poco lo que habíamos visto ese día [...] síntomas que habíamos visto en clase y eso"(E5).

Por otro lado, la inmersión en una unidad hospitalaria y su relación cercana sin bata con personas ingresadas en la unidad, le ayudó a desarrollar habilidades de comunicación y actitudes empáticas: "Cada día me sentía más segura" (E7), "Los pacientes entraban, 
salían $[\ldots]$ no sabíamos que decirles para que se uniesen al taller [...] luego ya mejor" (E8), "Creo que me ayudará en mis prácticas [...] me ayudará a que me implique más con los pacientes" (E4).

El aprendizaje y su inmersión en el funcionamiento del voluntariado de la unidad les permitió interactuar en mayor medida con las personas ingresadas y modificar sus ideas estigmatizantes por actitudes más positivas. Por ejemplo, una entrevistada dijo: "Sentí un poco de miedo [...] pero la segunda vez superbien, iba contenta y sin nervios de saber lo que me iba a encontrar [...] Creo que mi opinión ha cambiado para bien" (E4). "Te llevas muy buena experiencia, realmente ves que son personas $[. .$.$] que no hay nada malo en estar alli'"$ (E1), "Las personas con enfermedad mental se merecen respeto como todos" (E8). "Después de haber hecho el voluntariado creo que es trabajar como en una unidad normal y corriente" (E4). Incluso: "Siento que podría trabajar allí" (E6).

Del mismo modo, algún mito, como el de la agresividad asociada a las personas con enfermedad mental también fue desmontado: "Creo que puede haber gente agresiva en cualquier otra planta [...] Esa idea que tienes con prejuicios se ha ido modificando y ya tienes tu idea personal [...] que ha cambiado" (E2). "Al estar más en contacto ves que no pasa nada. Me di cuenta de que las personas ingresadas reaccionan como cualquier otra. Las sesiones que hemos ido haciendo también me han ayudado"(E6).

Las entrevistadas percibieron que su colaboración en la UHP había ayudado, de un modo u otro, de forma positiva a los pacientes. Remarcaron el entretenimiento y la oportunidad de conversar y expresar emociones que se ofreció a los pacientes ingresados en la unidad: "A mí me gusta mucho ayudar y creo que los pacientes se entretuvieron $[\ldots]$ y que luego lo agradecieron que hubiéramos ido" (E5). Porque según otra entrevistada (E7): "Estaban allí encerrados, aburridos, y cuando llegábamos a lo mejor bajábamos al patio a jugar a futbolín o al ping-pong y veías cómo sonreían”. Para otra estudiante (E8), el poder: "Llamarlos por su nombre, hacerles sentir ellos mismos, salir a pasear por fuera del hospital a tomar el aire [...] a mí me dio la sensación de que estaban a gusto con nosotras, que confiaban en nosotras, que se reían y nos contaban cosas [...] El primer día cuando llegamos participamos en una actividad de grupo que estaba haciendo el psicólogo y el enfermero que se llamaba 'Buenos días', y bueno, nos presentamos y básicamente escuchamos
[...] pero cuando acabaron les daban como un desayuno, entonces ahí empezamos a hablar de manera muy normal, como en una cafetería".

Todas las estudiantes de enfermería valoraron la experiencia como positiva, considerando que les había aportado conocimientos que en sus prácticas obligatorias no hubieran o habían podido alcanzar, como por ejemplo la comunicación con los pacientes. Una alumna dijo: "En las prácticas tienes que fijarte en otras cosas $[\ldots]$ solo ponía inyectables, no hice ninguna terapia de grupo, ni hablaba con los pacientes más allá de lo justo para la consulta" (E8).

"En las prácticas clínicas al tener el tiempo más limitado observas el ambiente y el funcionamiento de la unidad, mientras que, en el voluntariado, pude bajar con ellos al patio y tuve más contacto con ellos"(E9).

\section{Discusión}

Este estudio describe las principales experiencias de las estudiantes de enfermería voluntarias en una unidad de hospitalización psiquiátrica.

Actualmente es tendencia en la literatura sobre educación de enfermería evaluar cómo diferentes intervenciones educativas ayudan a moldear las características estigmatizantes en los estudiantes, centrándose en el alumno y maximizando su periodo teórico como el de prácticas clínicas ${ }^{12,13}$. Sin embargo, con esta propuesta buscamos una estrategia extracurricular. Nuestra programa de voluntariado se basó en las recomendaciones de Corrigan et al..$^{14}$, quienes señalaron tres aspectos a tener en cuenta para cambiar las actitudes hacia aquellos que experimentan problemas de salud mental: la educación teórica, el contacto y la objeción.

La mayoría de los estudiantes de enfermería, debido a su escasa experiencia vital, poseen limitada experiencia personal con personas que padecen algún trastorno mental grave al iniciar su formación teórico-práctica. Con el propósito de favorecer espacios de encuentro, de promover el papel de los usuarios en la educación y disminuir el estigma hacia las personas con enfermedad mental, se ofertó esta estrategia educativa en línea con los beneficios reportados en la revisión de Brenda Happell ${ }^{15}$.

Esta tendencia a la reducción de actitudes negativas de nuestro estudio, plantea preguntas sobre las expe- 
riencias educativas del alumnado y exige una investigación exhaustiva de los factores que contribuyen a esa direccionalidad y sobre las diferentes estrategias de entrenamiento, para identificarlas y plasmarlas en el programa curricular del Grado de enfermería mediante un diseño cualitativo más amplio.

\section{Limitaciones}

Las limitaciones del presente estudio están relacionadas con el tamaño reducido de la muestra representado por una única cohorte de una única facultad. Futuras investigaciones deben esforzarse por reclutar a un mayor número de estudiantes de más de una institución, así como aclarar si la participación fue determinada por alguna motivación personal o actitudes más positivas debido a la existencia de características personales pertenecientes a estudiantes de enfermería, o debido a su línea de base circunstancias tales como bienestar emocional o físico, niveles de confianza, ansiedad o empatía.

\section{Conclusiones}

La participación en estrategias educativas como voluntariado en la Unidad de Pacientes Intensivos de Salud Mental, complementaria a programas de pregrado y prácticas clínicas, permite a los estudiantes de Enfermería desarrollar actitudes más diversificadas y positivas hacia las enfermedades mentales y las personas con problemas de salud mental.

El voluntariado como estrategia educativa tuvo una influencia positiva en las actitudes de los estudiantes de enfermería como resultado del contacto directo con personas con trastornos mentales agudos. Se necesita el apoyo de maestros y enfermeras de salud mental que actúen como mentores en la unidad, para acompañarlos y guiarlos en situaciones que generan inseguridad.

\section{Bibliografía}

1. Aznar-Lou, I., Serrano-Blanco, A., Fernández, A., Luciano, J. V, \& Rubio-Valera, M.. Attitudes and intended behaviour to mental disorders and associated factors in catalan population, Spain: Cross-sectional population-based survey. BMC Public Health, 2016;16(1), 1-12. https://doi.org/10.1186/s12889-016-2815-5

2. Loubat, M., Lobos, R., \& Carrasco, N. Estigmatización de la persona con esquizofrenia y consecuencias para el proceso de rehabilitación: un estudio en profesionales de la salud mental. Límite. Revista Interdisciplinaria de Filosofía y Psicología. 2017;12(39), 15-25. Retrieved from:

http://limite.uta.cl/index.php/limite/article/viewFile/167/203
3. Chadwick, L., \& Porter, J. An Evaluation of the Effect of a Mental Health Clinical Placement on the Mental Health Attitudes of Student Nurses. Nursing and Health ,2014;2(3), 57-64. https://doi.org/10.13189/nh.2014.020301

4. Fresán, A., Robles, R., Cota, M., Berlanga, C., Lozano, D., \& Tena, A. Actitudes de mujeres estudiantes de psicología hacia las personas con esquizofrenia: Relación con la percepción de agresividad y peligrosidad. Salud Mental. 2012;35(3), 215-223.

5. Schafer, T., Wood, S., \& Williams, R. A survey into student nurses' attitudes towards mental illness: Implications for nurse training. Nurse Educ Today, 2011;31(4), 328-332. https://doi.org/10.1016/j.nedt.2010.06.010

6. Song, E. The Nursing Students' Experience of Psychiatric Practice in South Korea. Arch Psychiatr Nurs, 2015; 29 (5), 355-360. https://doi.org/10.1016/j.apnu.2015.06.005

7. Happell, B. The importance of clinical experience for mental health nursing - Part 1: Undergraduate nursing students' attitudes, preparedness and satisfaction. Int J Ment Health Nurs. 2008a; 17(5), 326-332. https://doi.org/10.1111/j.1447-0349.2008.00555.x

8. Happell, B. The importance of clinical experience for mental health nursing - Part 2: Relationships between undergraduate nursing students' attitudes, preparedness, and satisfaction. Int J Ment Health Nurs, 2008b; 17(5), 333-340. https://doi.org/10.1111/j.1447-0349.2008.00556.x

9. Patterson, C., Moxham, L., Brighton, R., Taylor, E., Sumskis, S., Perlman, D., Hadfield, L. Nursing students' reflections on the learning experience of a unique mental health clinical placement. Nurse Educ Today, 2016; 46, 94-98. https://doi.org/10.1016/j.nedt.2016.08.029

10. Abela, J. A. Las técnicas de Análisis de Contenido: una revisión actualizada. Fundación Centro de Estudios Andaluces, 2002; 1-34. https://doi.org/10.2307/334486

11. Guba, E. G., \& Lincoln, Y. S. (n.d.). Competing Paradigms in Qualitive Research.

12. Henderson, S., Happell, B. \& Martin, T. So what is so good about clinical experience: A mental health nursing perspective? Nurse Educ Pract, 2007; 7, 164-172

13. Hunter, L., Weber, T., Shattel, M. \& Harris, B. Nursing student's attitudes about psychiatric mental health nursing. Issues Ment Health Nurs, 2015; 36,29-34

14. Corrigan, P. W., Green, A., Lundin, R., Kubiak, M. \& Penn, D. Familiarity with and social distance from people with serious mental illness. Schizophrenia Bulletin, 2001;27, 219-226

15. Happell, B., Platania-Phung, C., Byrne, L., Wynaden, D., Marti, G. \& Harri, S. Consumer participation in nurse education: A national survey of Australian universities. Int J Ment Health Nurs, 2015;24. 95-103. doi:10.1111/inm.12111 